\title{
Dissipative feedback does not improve the optimal resolution of incoherent force detection
}

To the Editor - In 2012, Gavartin et al. reported a dissipative feedback scheme for an integrated optomechanical system ${ }^{1}$. The authors focus on detecting an incoherent force acting on a thermally driven resonator, and argue that dissipative feedback "can substantially improve the incoherent force resolution of nano- or micromechanical systems" and "enabling a significant decrease of measurement time and thus extending the range of applications for small-scale transducers."

However, in general, stationary linear feedback cannot improve the signalto-noise ratio of a force measurement, regardless of whether the force to be detected is coherent or incoherent. This is because feedback modifies the response of the resonator to both force signal and force noise in the same way. This concept is known to researchers involved in force detection experiments such as those that use gravitational wave detectors $^{2}$, atomic force microscopes ${ }^{3}$ and optomechanical systems ${ }^{4}$.

The results of Gavartin et al. seem to contradict this statement. In this respect, we observe that the force resolution depends on the specific estimator that is implemented. Gavartin et al. use an estimator based on the bare resonator energy (equation (4) in ref. 1), and conclude, coherently, that the force resolution is improved by feedback because of a shortening of the correlation time.
However, energy is not the only possible estimator, and it is definitely not the most efficient. An optimal method for such a problem is well known in data processing and is based on Wiener's theory of optimal filtering. For instance, its application to the specific problem of detecting an incoherent force acting on a resonator is discussed in ref. 5.

The optimal filter can be implemented off-line in most resonator-based systems without the need for additional hardware. It is essentially the product of an inverting filter and a bandpass filter. The inverting filter removes the narrowband resonator dynamics by converting the measured displacement into an equivalent force, whereas the bandpass filter minimizes the contribution of wideband detection noise. The correlation time of filtered data $\tau_{\mathrm{f}}$ is determined by the bandwidth over which the resonator noise overcomes the wideband detection noise, which is often (as in the work of Gavartin et al.) much larger than the intrinsic resonator bandwidth. Remarkably, $\tau_{\mathrm{f}}$ does not change when the actual resonator correlation time $\tau$ is modified by a dissipative feedback. As a consequence, the force resolution, which is obtained by time-averaging the filtered data, is also feedbackindependent. Actually, it can be shown that the optimal force resolution for a given averaging time depends only on the signalto-noise ratio ${ }^{5}$.
We note that the technique proposed by Gavartin et al., based on simple energy-averaging with a feedback-assisted reduction of the effective resonator time constant, can be considered as a way to approximate the optimal filter in hardware. However, it provides no additional improvement as soon as optimal filtering is applied.

In conclusion, stationary dissipative feedback does not improve the resolution of incoherent force detection, provided that appropriate data processing is implemented.

References

1. Gavartin, E., Verlot, P. \& Kippenberg, T. J. Nature Nanotech. 7, 509-514 (2012).

2. Oide, K., Hirakawa, H. \& Fujimoto, M. K. Phys. Rev. D 20, 2480-2483 (1979).

3. Mertz, J., Marti, O. \& Mlynek, J. Appl. Phys. Lett. 62, 2344-2346 (1993).

4. Vitali, D., Mancini, S. \& Tombesi, P. Phys. Rev. A 64, 051401 (2001).

5. Astone, P., Bonifazi, P. \& Pallottino, G. V. Rev. Sci. Instrum. 61, 3899-3903 (1990).

A. Vinante ${ }^{1 \star}$, M. Bonaldi ${ }^{2}$, F. Marin ${ }^{3}$ and J-P. Zendri ${ }^{4}$

${ }^{1} \mathrm{FBK}$, Institute of Photonics and

Nanotechnology, I-38123 Povo (TN), Italy,

${ }^{2}$ Institute of Materials for Electronics and

Magnetism, Nanoscience-Trento-FBK Division, I-38123 Povo (TN), Italy, ${ }^{3}$ Dipartimento di

Fisica, Universitá di Firenze, I-50019 Sesto

Fiorentino (FI), Italy, ${ }^{4}$ INFN, Sezione di Padova,

I-35131 Padova, Italy.

*e-mail: anvinante@fbk.eu 